\title{
Stability of Uncertain 2-D Discrete Systems in Presence of Generalized Overflow Nonlinearities
}

\author{
Suchitra Pandeya, Siva Kumar Tadepalli, V. Krishna Rao Kandanvli, Abhilav Vishwakarma
}

\begin{abstract}
Stability analysis of two-dimensional (2-D) discontinuous systems with generalized overflow nonlinear effects is considered in this work. The 2-D models considered are the well-known Fornasini Marchesini Second Local State-Space (FMSLSS) model and the Roesser model. The effect of uncertainties and interim-like variable time-delays on the system is also examined in the study. Using reciprocally convex approach we provide stability criteria which is organized as matrix inequalities. Numerical illustrations are given to demonstrate the applicability of the results.
\end{abstract}

Keywords : Delay-dependent stability criterion; Finite wordlength effect; Lyapunov stability; Delayed system; Uncertain system.

\section{INTRODUCTION}

Various physical systems can be modeled as two-dimensional (2-D) systems. 2-D models are needed as many times the one-dimensional (1-D) models are unable to capture the dynamics of the system. Such systems include image processing, monitoring and control of sensor networks, heat diffusion system (Xu \& Yu, 2009), thermal processes (Tadepalli, Kandanvli, \& Kar, 2015, 2016), iterative learning control (Tadepalli et al., 2015) etc. The 2-D modeling of physical systems has gained a lot of interest due to the two popular models Roesser (Roesser, 1975) and Fornasini Marchesini Second Local State-Space (FMSLSS) (Fornasini \& Marchesini, 1978).

During the implementation of discrete-time feedback systems on a fixed-point digital hardware instabilities (sort of limit cycles) might appear in the system owing to the overflow of data. In order to avoid overflow, overflow correction techniques such as saturation, zeroing, two's complement and triangular are employed. Since the finite wordlength implementation of digital systems results in systems that are inherently nonlinear, therefore the investigation of asymptotic stability of such systems has attracted the attention of several researchers (Chen, 2010a, 2010b; Dey \& Kar, 2011a, 2011b; Kandanvli \& Kar, 2008, 2012; D. Liu, 1998).

Revised Manuscript Received on September 22, 2019

* Correspondence Author

Suchitra Pandeya *, Department of Electronics \& Telecommunication Engineering, Bhilai Institute of Technology, Durg, 491001, India.

Siva Kumar Tadepalli, Department of Electronics Engineering, National Institute of Technology, Uttarakhand, 246174, India

V. Krishna Rao Kandanvli, Department of Electronics \& Communication Engineering, Motilal Nehru National Institute of Technology, Allahabad, 211004, India

Siva Kumar Tadepalli, Department of Electronics \& Telecommunication Engineering, Bhilai Institute of Technology, Durg, 491001, India
In general, practical systems involve uncertainties and time-lags which are also sources of instabilities in systems. Therefore, analysis of discontinuous systems subjected to finite wordlength nonlinear effects, uncertainties and time-lags from stability viewpoint, is a significant problem. A globally asymptotically stable, time-lag independent condition was reported for an uncertain discontinuous-time system with constant state-delays and influenced by generalized overflow nonlinear effects in (Kandanvli \& Kar, 2008). In, (Kandanvli \& Kar, 2012) delay-dependent theorem has been developed for 1-D discontinuous systems involving generalized overflow nonlinear effects, uncertainties and variable time-lags. In (Dey \& Kar, 2011a) delay-independent condition for stability has been evaluated for 2-D systems characterized by the FMSLSS model under the simultaneous influence of generalized overflow nonlinear effects, uncertainties and delays.

Motivated by the above results, this paper considers the problem of developing time-lag dependent global asymptotic stability criteria for uncertain discontinuous-time systems with interim-like variable time-lag in the state with a generalized characterization of the overflow nonlinearities. The different sections of the paper are: In Section 2 description of the generalized overflow nonlinearities and the lemmas used are provided. In Section 3 we present the significant results of the paper. Examples have been presented in Section 4 to depict the significance of the developed criteria.

Notations used all along the paper are as follows:

$$
\begin{aligned}
& \mathbb{R}^{\mathrm{pxq}} \quad \text { set of } \mathrm{p} \mathrm{x} \mathrm{q} \mathrm{real} \mathrm{matrices} \\
& \mathbb{R}^{\mathrm{p}} \quad \text { set of } \mathrm{p} \times 1 \text { real vectors } \\
& \text { 0 null matrix or null vector of suitable dimension } \\
& \text { I identity matrix of appropriate dimension } \\
& \boldsymbol{I}_{n} \quad \text { unit matrix of order } n \\
& T \quad \text { superscript ' } T \text { ' specifies transpose } \\
& \boldsymbol{B}>0 \quad \boldsymbol{B} \text { is positive definite symmetric matrix } \\
& \mathbf{B}_{1} \oplus \mathbf{B}_{2} \text { direct sum, i.e. } \mathbf{B}=\left[\begin{array}{cc}
B_{1} & 0 \\
0 & B_{2}
\end{array}\right]
\end{aligned}
$$

\section{GENERALIZED OVERFLOW NONLINEARITIES}

The nonlinearity $f_{i} y_{i}(\gamma)(i=1,2, \ldots, n)$ under consideration is the generalized overflow characteristic given by 


$$
\left.\begin{array}{c}
\mathcal{L} \leq f_{i}\left(y_{i}(\gamma)\right) \leq \mathcal{L}_{1}, y_{i}(\gamma)>1 \\
f_{i}\left(y_{i}(\gamma)\right)=y_{i}(\gamma),-1 \leq y_{i}(\gamma) \leq 1 \\
-\mathcal{L}_{2} \leq f_{i}\left(y_{i}(\gamma)\right) \leq-\mathcal{L}_{y}(\gamma)<-1
\end{array}\right\}, i=1,2,3, \ldots n
$$

where $-1 \leq \mathcal{L} \leq 1_{0} \mathcal{L} \leq \mathcal{L}_{1} \leq 1$ and $\mathcal{L} \leq \mathcal{L}_{2} \leq 1$. One can observe that $\mathcal{L}=\mathcal{L}_{1}=\mathcal{L}_{2}=1, \mathcal{L}=\mathcal{L}_{1}=\mathcal{L}_{2}=0$, $\mathcal{L}=-1_{x} \mathcal{L}_{1}=\mathcal{L}_{2}=1$ and $\mathcal{L}=-1_{2} \mathcal{L}_{1}=\mathcal{L}_{2}=1$ represents saturation, zeroing, triangular and two's complement overflow arithmetic, respectively.

Generalized overflow nonlinearities occur during the limited wordlength (or fixed-point) realization of discrete systems. In the previous studies a time-lag dependent stability condition for 2-D discontinuous systems involving generalized overflow nonlinear effects has not been provided. This work finds significance in the light that the models considered for the purpose of study are beneficial for representing a extensive variety of practical systems such as heat diffusion, thermic energy processes, river pollution modeling, seismorgraphic data processing, wireless sensor networks, iterative learning control, etc.

To prove our main results the lemmas that play a consequential role are:

\section{Lemma 2.1:}

(J. Liu \& Zhang, 2012; Park, Ko, \& Jeong, 2011) For any matrices $\boldsymbol{R}_{x} \boldsymbol{S}$, vectors $\xi_{1^{x}} \xi_{2^{x}}$ and real numbers $\alpha_{1} \geq 0, \alpha_{2} \geq 0$ satisfying

$\left[\begin{array}{ll}R & S \\ * & R\end{array}\right] \geq 0, \quad \alpha_{1}+\alpha_{2}=1$

$\xi_{i}=0$ if $\alpha_{i}=0, \quad i=1,2$

one has

$-\frac{1}{\mathrm{x}_{1}} \xi_{1}{ }^{T} R \xi_{1}-\frac{1}{\mathrm{x}_{2}} \xi_{2}{ }^{\mathrm{T}} R \xi_{2} \leq-\left[\begin{array}{l}\xi_{1} \\ \xi_{2}\end{array}\right]^{T}\left[\begin{array}{ll}R & S \\ * & R\end{array}\right]\left[\begin{array}{l}\xi_{1} \\ \xi_{2}\end{array}\right]$

Lemma 2.2:

(Boyd, El Ghaoui, Feron, \& Balakrishnan, 1994; Xie, Fu, \& de Souza, 1992) Let $\boldsymbol{\Sigma}_{s} \boldsymbol{\Gamma}_{\mathrm{v}} \boldsymbol{F}$, and $\boldsymbol{M}$ be existent matrices of suitable sizes with $\boldsymbol{M}$ fulfilling $\boldsymbol{M}=\boldsymbol{M}^{T}$, then

$$
\boldsymbol{M}+\mathbf{\Sigma F \Gamma}+\Gamma^{T} \mathbf{F}^{T} \mathbf{\Sigma}^{T}<0
$$

$\forall F^{T} \boldsymbol{F} \leq \boldsymbol{I}_{x}$ if and only if there exists a scalar $E>0$ such that

$$
M+\epsilon^{-1} \mathbf{\Sigma} \mathbf{\Sigma}^{T}+e \Gamma^{T} \Gamma<0
$$

Lemma 2.3:

(Dey \& Kar, 2011b) A positive definite symmetric matrix $\boldsymbol{C}=\left[c_{i j}\right] \in \mathbb{R}^{n \times n}$ characterized by

$$
\begin{array}{ll}
c_{i i}=g_{i}+\sum_{j=1, j \neq i}\left(\alpha_{i j}+\beta_{i j}\right), & i=1,2, \ldots, n, \\
c_{i j}=c_{j i}+\frac{(1+L)}{2}\left(\alpha_{i j}-\beta_{i j}\right), & i, j=1,2, \ldots, n(i \neq j) .
\end{array}
$$

$$
\alpha_{i j}=\alpha_{j i}>0, \beta_{i j}=\beta_{j i}>0, \quad i_{s} j=1,2, \ldots, n(i \neq j),
$$

$g_{\mathrm{i}}>0, \quad i=1,2 \ldots \ldots n$.

(2.6d)

satisfies

\section{$y^{T}(\gamma) C y(\gamma)-f^{T}(y(\gamma)) C f(y(\gamma)) \geq 0$}

We now put forward the main results of this paper.

\section{MAIN RESULTS}

3.1. Stability criteria for 1-D uncertain discontinuous-time systems involving generalized overflow nonlinear effects

The system to be examined is characterized as follows

$$
\begin{aligned}
\boldsymbol{x}(\gamma+1) & =f(y(\gamma)) \\
& =\left[f_{1}\left(\gamma_{1}(\gamma)\right) f_{2}\left(\mathrm{Y}_{2}(\gamma)\right) \quad \ldots f_{n}\left(Y_{n}(\gamma)\right)\right]^{T}
\end{aligned}
$$

$$
y(\gamma)=(A+\Delta A) x(\gamma)+\left(A_{d}+\Delta A_{d}\right) x(\gamma-d(\gamma)
$$

$$
\begin{aligned}
& =\left[\begin{array}{llll}
y_{1}(\gamma) & y_{2}(\gamma) & \ldots & y_{n}(\gamma)
\end{array}\right]^{T} \\
& x(\gamma)=\varphi(\gamma) \forall \gamma=-d_{2^{x}}-d_{2}+1_{x \ldots y} 0
\end{aligned}
$$

where $\boldsymbol{x}(\boldsymbol{\gamma}) \in \mathbb{R}^{n}$ is the state vector; $\boldsymbol{A}_{v} \boldsymbol{A}_{d} \in \mathbb{R}^{n \times n}$ are the known eternal matrices; $\Delta A_{x} \Delta A_{d} \in \mathbb{R}^{n \times n}$ are the known matrices symbolizing uncertainties in the state matrices; $\varphi(\gamma) \in \mathbb{R}^{\mathrm{n}}$ is the primary state at time $\gamma ; f(\cdot)$ characterizes the generalized overflow nonlinear effects; and $d(\gamma)$ is a variable time-lag satisfying

$$
d_{1} \leq d(r) \leq d_{2}
$$

where $d_{1}$ and $d_{2}$ are familiar positive integers defining the lower and upper time-lag limits, respectively. The uncertainties are presumed as

\section{$\left[\Delta A \Delta A_{d}\right]=H F\left[E_{0} E_{1}\right]$}

(3.10a)

where $H \in \mathbb{R}^{n \times p}$ and $E_{0}, E_{1} \in \mathbb{R}^{q \times n}$ are the matrices that are known and constant and $\boldsymbol{F} \in \mathbb{R}^{n \times p}$ is an unknown matrix which suffices

\section{$\boldsymbol{F}^{T} \boldsymbol{F} \leq \boldsymbol{I}_{x}$}

(3.10b)

The above uncertainty structure (3.10) has widespread usage in filtering applications and robust control for systems with uncertainties (Dey \& Kar,2011a; Kandanvli \& Kar, 2012; Xie et al., 1992). 
A time-lag dependent LMI-based stability criterion is now presented in the form of Theorem 3.1.

\section{Theorem 3.1:}

Given two integers $d_{1}$ and $d_{2}$ satisfying $0<d_{1}<d_{2}$, the system represented by (3.8)-(3.10) is globally asymptotically stable if there exist matrices $\boldsymbol{P}>\mathbf{0}, \boldsymbol{Q}_{\mathrm{i}}>\mathbf{0}(i=1,2,3), Z_{1}>\mathbf{0}, \boldsymbol{Z}_{2}>\mathbf{0}$, a matrix $\boldsymbol{S}$ with compatible dimensions, and positive scalars $\epsilon_{0,}, \epsilon_{1}, g_{i}(i=1,2, \ldots, n), \alpha_{i j}, \beta_{i j}(i, j=1,2, \ldots n(i \neq j))$ such that

$\Theta=\left[\begin{array}{cc}Z_{2} & S \\ * & Z_{2}\end{array}\right]>0$

$\left[\begin{array}{ccccccc}\Xi_{11}+\epsilon_{0} E_{0}{ }^{T} E_{0} & 0 & Z_{1} & 0 & \Xi_{15} & A^{T} C & 0 \\ * & \Xi_{22}+\epsilon_{1} E_{1}{ }^{T} E_{1} & Z_{2}-S^{T} & Z_{2}-S & 0 & A_{d}^{T} C & 0 \\ * & * & -Q_{1}-Z_{1}-Z_{2} & S & 0 & 0 & 0 \\ * & * & * & -Q_{2}-Z_{2} & 0 & 0 & 0 \\ * & * & * & * & \Xi_{55} & 0 & 0 \\ * & * & * & * & * & -C & C H \\ * & * & * & * & * & * & -\epsilon I\end{array}\right]<0$

where

$\mathbf{E}_{11}=P+\sum_{i=1}^{a} Q_{i}+d_{12} Q_{a}-\Xi_{15}-Z_{1}$

$\mathrm{E}_{15}=-\left(d_{1}^{2} Z_{1}+d_{12}^{2} Z_{2}\right)$

(3.14)

$\mathrm{E}_{22}=-Q_{\mathrm{a}}-2 Z_{2}+S+S^{T}$

(3.15)

$\mathrm{E}_{55}=\boldsymbol{P}-\mathrm{E}_{15}-\boldsymbol{C}$

(3.16)

$d_{12}=d_{2}-d_{1}$

(3.17)

and $\boldsymbol{C}$ is given by (2.6).

Proof

Select a LKF (Huang \& Feng, 2010; Tadepalli, Kandanvli, \& Kar, 2014; Tadepalli \& Kandanvli, 2016) as

$V(x(\gamma))=x^{T}(\gamma) P x(\gamma)+\sum_{\mu=\gamma-d_{1}}^{\gamma-1} x^{T}(\mu) Q_{1} x(\mu)+\sum_{\mu=\gamma-d_{2}}^{\gamma-1} x^{T}(\mu) Q_{2} x(\mu)$

$+d_{12} \sum_{\theta=-d_{2}+1}^{-d_{1}} \sum_{v=\Upsilon \Upsilon \Upsilon}^{\gamma-1+\theta} \eta^{T}(v) Z_{2} \eta(v)$

(3.18)

where

$\eta(\gamma)=x(\gamma+1)-x(\gamma)=f(y(\gamma))-x(\gamma)$

Finding the finite difference of LKF down the flight path of the system (3.8), as follows $\Delta V(x(r))=V(x(r+1))-V(x(r))$ $+\sum_{v=-d_{2}}^{-d_{1}} \sum_{\mu=\gamma+q}^{\mathrm{\gamma}-1} x^{T}(\mu) Q_{2} x(\mu)+d_{1} \sum_{\theta=-d_{1}+1}^{0} \sum_{v=\gamma-1+\theta}^{\mathrm{\gamma}-1} \eta^{T}(v) Z_{1} \eta(v)$

$=f^{T}(y(\gamma)) P f(y(\gamma))-x^{T}(\gamma) P x(\gamma)+x^{T}(\gamma) Q_{1} x(\gamma)-x^{T}\left(\gamma-d_{1}\right) Q_{1} x\left(\gamma-d_{1}\right)+x^{T}(\gamma) Q_{2} x(\gamma)$ $-x^{T}\left(\gamma-d_{2}\right) Q_{2} x\left(\gamma-d_{2}\right)+\left(d_{12}+1\right) x^{T}(\gamma) Q_{3} x(\gamma)$

$$
\begin{aligned}
& -\sum_{\mu=\gamma-d_{2}}^{\gamma-d_{1}} x^{T}(\mu) Q_{3} x(\mu)+d_{1}{ }^{2} \eta^{T}(\gamma) Z_{1} \eta(\gamma)+d_{12}{ }^{2} \eta^{T}(\gamma) Z_{2} \eta(\gamma) \\
& -d_{1} \sum_{\mu=\gamma-d_{2}}^{\gamma-1} \eta^{T}(\mu) Z_{1} \eta(\mu)-d_{12} \sum_{\mu=\gamma-d_{2}}^{\gamma-d_{1}-1} \eta^{T}(\mu) Z_{2} \eta(\mu)
\end{aligned}
$$

Using Jensen's Inequality for discrete-time systems, the following relation is obtained

$$
\begin{gathered}
-d_{1} \sum_{\mu=\gamma-d_{1}}^{\mathrm{\gamma}-1} \boldsymbol{\eta}^{T}(\mu) Z_{1} \boldsymbol{\eta}(\mu) \leq-\sum_{\mu=\mathrm{\gamma}-d_{1}}^{\mathrm{\gamma}-1} \boldsymbol{\eta}^{T}(\mu) Z_{1} \sum_{\mu=-d_{1}}^{\gamma-1} \eta(\mu) \\
=-\left[x^{T}(\gamma)-x^{T}\left(\gamma-d_{1}\right)\right] Z_{1}\left[x(\gamma)-x\left(\gamma-d_{1}\right)\right]
\end{gathered}
$$

Further, employing Jensen's inequality and Lemma 2.1, the following relation can be obtained if there exists a matrix $S$ such that (11) holds.

$-d_{12} \sum_{\mu=-d_{2}}^{\gamma-d_{1}-1} \eta^{T}(\mu) Z_{2} \eta(\mu) \leq-\frac{1}{\frac{\left(d(\gamma)-d_{1}\right)}{d_{12}}} \gamma_{1}^{T}(\gamma) Z_{2} \gamma_{1}(\gamma)-\frac{1}{\frac{\left(d_{2}-d(\gamma)\right)}{d_{12}}} \gamma_{2}^{T}(\gamma) Z_{2} \gamma_{2}(\gamma)$

$$
\leq-\left[\begin{array}{l}
\gamma_{1}(\gamma) \\
\gamma_{2}(\gamma)
\end{array}\right]^{T} \theta\left[\begin{array}{l}
\gamma_{1}(\gamma) \\
\gamma_{2}(\gamma)
\end{array}\right]
$$

where

$$
\begin{aligned}
& \gamma_{1}(\gamma)=x\left(\gamma-d_{1}\right)-x(\gamma-d(\gamma)) \\
& Y_{2}(\gamma)=x(\gamma-d(\gamma))-x\left(\gamma-d_{2}\right)
\end{aligned}
$$

Note that

$$
-\sum_{\mu=\Upsilon-d_{2}}^{\gamma-d_{1}-1} x^{T}(\mu) Q_{\mathrm{a}} x(\mu) \leq-x^{T}(\gamma-d(\gamma)) Q_{\mathrm{a}} x(\gamma-d(\gamma)) \text {. }
$$

Utilizing expressions (3.20)-3.24), the following inequality is obtained

$$
\Delta V(x(\gamma)) \leq \xi^{T}(\gamma) \Psi_{1} \xi(\gamma)-\beta
$$

Where

$$
\beta=y^{T}(\gamma) C y(\gamma)-f^{T}(y(\gamma)) C f(y(\gamma))
$$

$$
\Psi_{1}=\left[\begin{array}{ccccc}
\mathbb{\Xi}_{11} \bar{A}^{T} \bar{C} & \bar{A}^{T} C \bar{A}_{d} & Z_{1} & 0 & \Xi_{15} \\
* & \bar{\Xi}_{22}+\bar{A}_{d}^{T} C \bar{A}_{d} & Z_{2}-S^{T} & Z_{2}-S & 0 \\
* & * & -Q_{1}-Z_{1}-Z_{2} & S & 0 \\
* & * & * & -Q_{2}-Z_{2} & 0 \\
* & * & * & * & \bar{\Xi}_{55}
\end{array}\right]
$$

$\bar{A}=A+\Delta A, \bar{A}_{d}=A_{d}+\Delta A_{d}$

and 


$$
\xi^{T}(\gamma)=\left[x^{T}(\gamma) x^{T}(\gamma-d(\gamma)) x^{T}\left(\gamma-d_{1}\right) x^{T}\left(\gamma-d_{2}\right) f^{T}(y(\gamma))\right]
$$

One can observe that for the nonlinear effects given by (2.1), the coefficient $\beta$ given in (3.26) is nonnegative (Kandanvli \& Kar, 2008; Dey \& Kar, 2011b). So, from (3.25), $\Delta V(x(\gamma))<0$, only if $\vec{\Psi}_{1}<0$. Thus $\vec{\Psi}_{1}<0$ together with (3.11) are globally asymptotically stable and sufficient conditions for the system given by (3.8)-(3.10). Using Schur's complement $\Psi_{1}<0$ can be explicitly written as

$$
\left[\begin{array}{cccccc}
\Xi_{11} & 0 & Z_{1} & 0 & \Xi_{15} & \bar{A}^{T} C \\
* & \Xi_{22} & Z_{2}-S^{T} & Z_{2}-S & 0 & \bar{A}_{d}^{T} C \\
* & * & -Q_{1}-Z_{1}-Z_{2} & -Q_{2}-Z_{2} & 0 & 0 \\
* & * & * & * & 0 & 0 \\
* & * & * & * & \Xi_{55} & 0 \\
* & * & * & * & * & -C
\end{array}\right]<0
$$

Further, using (3.10a), and employing Lemma 2.2 one can easily arrive at Theorem 3.1. This completes the proof of Theorem 3.1.

\section{Remark 3.1:}

It is worth comparing the number of decision variables for the proposed Theorem 3.1 with Theorem 3 in (Kandanvli \& Kar, 2008). For a system with order $n$ the number of decision variables required for Theorem 3.1 is $6 n^{2}+2 n+1$ while for Theorem 3 in (Kandanvli \& Kar, 2008) it is $15 n^{2}+4 n+1$. Thus, Theorem 3.1 has the advantage of smaller number of decision variables in comparison with Theorem 3 of (Kandanvli \& Kar, 2008) while maintaining the same level of conservativeness.

Next, we extend the one-dimensional (1-D) results to the two-dimensional (2-D) case.

\subsection{Stability criterion for 2-D discrete uncertain systems represented by the Roesser model}

$x_{11}\left(x_{,} \lambda\right)=\left[\frac{x^{h}(K+1, \lambda)}{x^{v}(K, \lambda+1)}\right] \quad=f(y(x, \lambda))=\left[\frac{f^{h}\left(y^{h}(K, \lambda)\right)}{f^{v}\left(y^{v}(k, \lambda)\right)}\right]$

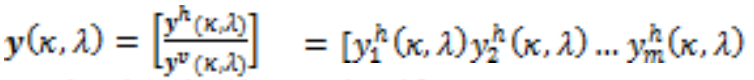

$$
\begin{aligned}
& \left.\| y_{1}^{V}\left(\kappa_{v} \lambda\right) y_{2}^{V}\left(\kappa_{z}, \lambda\right) \ldots y_{m}^{V}\left(\kappa_{v} \lambda\right)\right]
\end{aligned}
$$

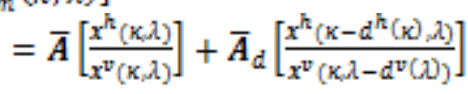$$
\text { (3.31b) }
$$

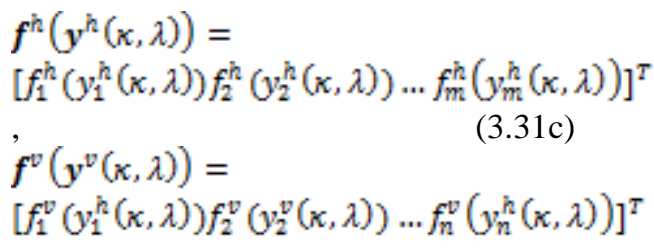$$
\kappa \geq 0, \lambda \geq 0
$$

(3.31e)

where
$\bar{A}=A+\Delta A_{s} \quad \bar{A}_{d}=A_{d}+\Delta A_{d}$.

$$
A=\left[\frac{A_{11}}{A_{21}} \mid \frac{A_{12}}{A_{22}}\right], A_{d}=\left[\frac{A_{d_{11}}}{A_{d_{21}}} \mid \frac{A_{d_{12}}}{A_{d_{22}}}\right]
$$

$$
\Delta A=\left[\frac{\Delta A_{11}}{\Delta A_{21}} \mid \frac{\Delta A_{12}}{\Delta A_{22}}\right], A_{d}=\left[\frac{\Delta A_{d_{11}}}{\Delta A_{d_{21}}} \mid \frac{\Delta A_{d_{12}}}{\Delta A_{d_{22}}}\right]
$$

$\kappa \in \mathbb{Z}_{+}$and $\lambda \in \mathbb{Z}_{+}$are the space coordinates forth the horizontal and vertical directions, respectively. The state vectors $\boldsymbol{x}^{\mathrm{h}}(\kappa, \lambda) \in \mathbb{R}^{\mathrm{m}}$ and $\boldsymbol{x}^{\mathrm{v}}(\kappa, \lambda) \in \mathbb{R}^{\mathrm{n}}$ disseminate information horizontally and vertically, respectively. The matrices

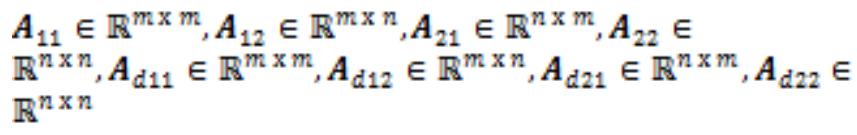
are the known coefficient matrices and $\Delta A_{11} \in \mathbb{R}^{m x m}{ }_{s} \Delta A_{12} \in \mathbb{R}^{m x{ }_{n}} \Delta A_{21} \in \mathbb{R}^{n x m}{ }_{0} \Delta A_{22} \in$

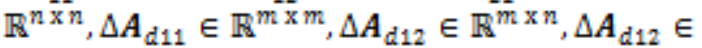
$\mathbb{R}^{\mathrm{nxm}}, \Delta A_{\text {d11 }} \in \mathbb{R}^{\mathrm{nxn}}$

are the matrices that are unknown and represent parameter uncertainties in the state matrices. $d^{\text {h }}(x)$ and $d^{\mathbb{D}}(\lambda)$ are variable time-lags along horizontal and vertical direction, respectively. Referring (Chen, 2010a), Let

$d_{1}^{h} \leq d^{h}(x) \leq d_{2}^{h}, \quad d_{1}^{D} \leq d^{v}(\lambda) \leq d_{2}^{v}$ (3.35)

where $d_{1}^{\text {ha }}$ and $d_{1}^{v}$ are positive integers that are constant and represent the lower time-lag limits along horizontal and vertical direction, respectively; $d_{2}^{\frac{1}{2}}$ and $d_{2}^{v}$ are positive constant integers symbolizing the upper time-lag limits along horizontal and vertical directions respectively, $f(v)$ represents the generalized overflow nonlinearities.

Let the parameter uncertainties that appear in the state matrices take the following form

\section{$\Delta A=H F E_{0}$}

(3.36a)

$\Delta A_{d}=H F E_{1}$

where

$$
\begin{aligned}
& H=\left[\begin{array}{l}
H_{m \times p}^{n} \\
H_{n x p}^{v}
\end{array}\right] \in \mathbb{R}^{(m+n) \times p} \\
& \boldsymbol{E}_{\mathrm{i}}=\left[\begin{array}{c}
\boldsymbol{E}_{\mathrm{i} q \mathrm{~h}}^{\mathrm{h}} \\
\boldsymbol{E}_{\mathrm{i} q \mathrm{~m}}^{v}
\end{array}\right] \in \mathbb{R}^{q x(m+n)}, i=0,1
\end{aligned}
$$

are constant matrices that are known and $F \in \mathbb{R}^{p \times q}$ is an unknown matrix which satisfies

$F^{T} F \leq I$.

It is assumed (Chen, 2010a; Xu, Nan, Zhang, Ou, \& Ni, 2013) that system in question has a limited set of boundary conditions, i.e., there exist two

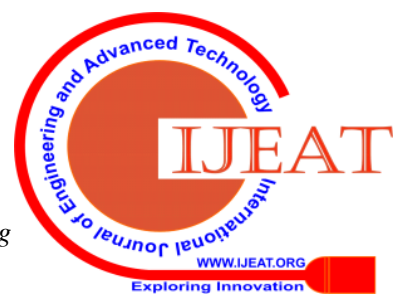


nonnegative integers $K$ and $L$ such that

$x^{h}(\kappa, \lambda)=v_{K \lambda,}, \forall 0 \leq \lambda<K, d_{2}^{h} \leq \kappa \leq 0$,

$x^{v}(\kappa, \lambda)=\varpi_{K \lambda}, \forall 0 \leq \kappa<L_{x} d_{2}^{v} \leq \lambda \leq 0$,

$v_{00}=\varpi_{00 \times}$

(3.37)

\section{Theorem 3.2:}

For given positive integers $d_{1}^{\text {h }}$ s $d_{2}^{\text {h }} d_{1}^{v}$ s $d_{2}^{v}$ satisfying $0<d_{1}^{\mathrm{h}_{\mathrm{h}}}<d_{2}^{\mathrm{h}}$ and $0<d_{1}^{\mathrm{V}}<d_{2}^{\mathrm{V}}$, the zero solution of the system described by (3.31)-(3.37) is globally asymptotically stable if there exist positive definite symmetric matrices

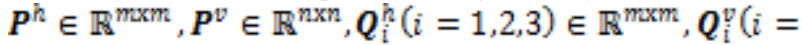
$1,2,3) \in \mathbb{R}^{n x n}{ }_{s} Z_{i}^{h}(i=1,2) \in \mathbb{R}^{\text {mxxm }}{ }_{v} Z_{i}^{v}(i=1,2) \in \mathbb{R}^{\text {nxn }}$ , matrices $S^{\text {h }}$ and $S^{v}$ with appropriate dimensions, scalars $\alpha_{i j}=\alpha_{i j}>0,=\beta_{i j}=\beta_{i j}>0, g_{i}>0, i_{s} j=1,2, \ldots, n(i \neq$ i) and $\in>0$ satisfying the LMIs

$\theta^{h}=\left[\begin{array}{cc}Z_{2}^{h} & S^{h} \\ * & Z_{2}^{h}\end{array}\right] \geq 0$

$$
\boldsymbol{\theta}^{v}=\left[\begin{array}{cc}
Z_{2}^{v} & S^{v} \\
* & Z_{2}^{v}
\end{array}\right] \geq 0
$$

$\left[\begin{array}{cc}M_{11} & \epsilon E_{0}{ }^{T} E_{1} \\ * & M_{22} \\ * & * \\ * & * \\ * & * \\ * & * \\ * & *\end{array}\right.$

$\mathbf{0}$

where

$M_{11}=-P+Q_{1}+Q_{2}+Q_{a}+d_{12} Q_{a}-M_{15}-Z_{1}+$ $\epsilon E_{0}^{T} E_{0}$

$M_{15}=-\left(d_{1}^{2} Z_{1}+d_{12}^{2} Z_{2}\right)$

(3.42)

$M_{22}=\epsilon E_{1}^{T} E_{1}-Q_{a}+2 Z_{2}+S^{T}+S$

(3.43)

$M_{55}=P+d_{1}^{2} Z_{1}+d_{12}^{2} Z_{2}-G$

(3.44)

$d_{12}^{h}=d_{2}^{h}-d_{1}^{h}$

(3.45)

$d_{12}^{V}=d_{2}^{V}-d_{1}^{D}$

(3.46)

and

$\boldsymbol{P}=\boldsymbol{P}^{\mathrm{h}} \oplus \boldsymbol{P}^{v}, \boldsymbol{Q}_{\mathrm{i}}=\boldsymbol{Q}_{\mathrm{i}}^{\mathrm{h}} \oplus \boldsymbol{Q}_{\mathrm{i}}^{\mathrm{V}}(i=1,2,3), \boldsymbol{Z}_{\mathrm{i}}=\boldsymbol{Z}_{\mathrm{i}}^{\mathrm{h}} \oplus$

$Z_{\mathrm{i}}^{\mathrm{v}}(i=1,2), G=G^{\mathrm{h}} \oplus \boldsymbol{G}^{v}$,

$S=S^{h} \oplus S^{v}, d_{1}=\left[\begin{array}{ll}d_{1}^{h} I_{m} & 0 \\ 0 & d_{1}^{V} I_{n}\end{array}\right], d_{12}=\left[\begin{array}{ll}d_{12}^{h} I_{m} & 0 \\ 0 & d_{12}^{V} I_{n}\end{array}\right]$

Proof

Consider a 2-D quardratic Lyapunov function (Tadepalli et al. 2015)

$V(x(x, \lambda))=V^{h}\left(x^{h}(x, \lambda)\right)+V^{v}\left(x^{D}\left(x_{x} \lambda\right)\right)$

where

$$
\begin{aligned}
& V^{h}\left(x^{h}(x, \lambda)\right)=x^{h^{T}}(x, \lambda) P^{h} x^{h}(x, \lambda)+\sum_{\pi=-d_{1}^{h}}^{-1} x^{h^{T}}(x+\pi, \lambda) Q_{1}^{h} x^{h}(x+\pi, \lambda)+\sum_{\pi=-d_{2}^{h}}^{-1} x^{h^{T}}(x+\pi, \lambda) Q_{2}^{h} x^{h}(x+\pi, \lambda) \\
& +\sum_{\rho=-d_{2}^{h} \pi=\rho}^{-d_{1}^{h}} \sum^{-1} x^{h^{T}}(x+\pi, \lambda) Q_{2}^{h} x^{h}(x+\pi, \lambda)
\end{aligned}
$$

$$
\begin{aligned}
& V^{v}\left(x^{v}(x, \lambda)\right)=x^{V^{T}}(x, \lambda) P^{v} x^{V}(x, \lambda) \sum_{\pi=-L_{1}^{v}}^{-1} x^{V^{T}}(x, \lambda+\pi) Q_{1}^{v} x^{v}(x, \lambda+\pi)
\end{aligned}
$$

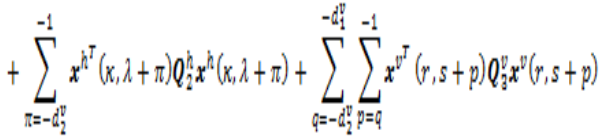

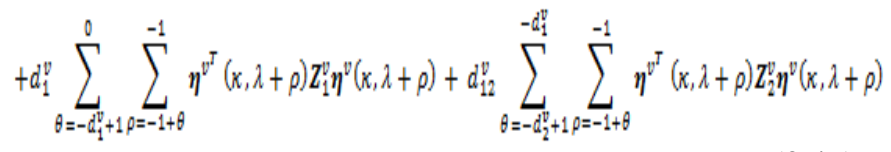

and

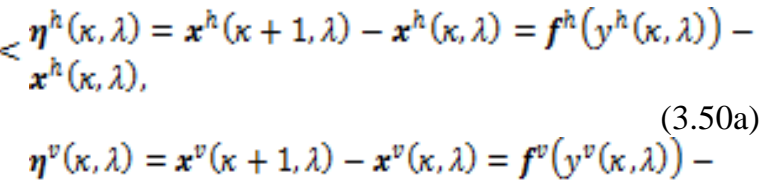

$\boldsymbol{x}^{\mathrm{D}}\left(\boldsymbol{\kappa}_{0} \lambda\right)$

Now, finding the finite difference of the LKF (3.47) along the system trajectories (3.31)-(3.37) and if one follows the steps similar to (3.21)-(3.30), can easily arrive at Theorem 3.2.

\section{Remark 3.2:}

Note that a time-lag dependent stability condition for 2-D systems characterized by the Roesser model involving generalized overflow nonlinear effects has not been previously studied.

For

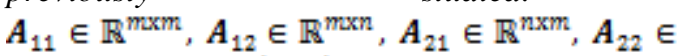
$\mathbb{R}^{n \times n}$ and $H \in \mathbb{R}^{(m+n) x p}$,

the cumulative count of scalar decision variables for Theorem 3.2 is given by $5\left(n^{2}+m^{2}\right)+3(n+m)+1$.

\subsection{Stability criterion for 2-D systems defined by FMSLSS model}

Let the system characteristics be defined by $\chi(\gamma+1, \delta+1)=f(\psi(\gamma, \delta))$,

$=\left[f_{1}\left(\psi_{1}(\gamma, \delta)\right) f_{2}\left(\psi_{2}(\gamma, \delta) \ldots f_{n}\left(\psi_{n}(\gamma, \delta)\right)\right]^{T}\right.$ (3.51a)

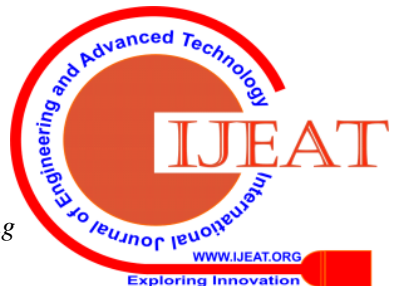


$\psi(\gamma, \delta)=\left(A_{1}+\Delta A_{1}\right) \chi(\gamma, \delta+1)+\left(A_{2}+\Delta A_{2}\right) \chi(\gamma+1, \delta)+\left(A_{d 1}+\Delta A_{d 1}\right) \chi(\gamma-\alpha(\gamma), \delta+1)$

$+\left(A_{d 2}+\Delta A_{d 2}\right) \chi(\gamma+1, \delta-\beta(\delta))$

$=\left[\psi_{1}(\gamma, \delta) \psi_{2}(\gamma, \delta) \quad \ldots \quad \psi_{n}(\gamma, \delta)\right]^{T}$

(3.51b)

where $\boldsymbol{f}^{(-)}$represents the generalized overflow nonlinearities, $\gamma \in \mathbb{Z}_{+}$and $\delta \in \mathbb{Z}_{+}$are horizontal and vertical coordinates, respectively; $\chi(y, \delta)) \in \mathbb{R}^{n}$ is the state local vector; $A_{1}, A_{2}, A_{d 1}, A_{d 2} \in \mathbb{R}^{n x n}$ are the known constant matrices; $\Delta A_{1}, \Delta A_{2}, \Delta A_{d 1}, \Delta A_{d 2} \in \mathbb{R}^{n \times n}$ are the known matrices that represent parametric uncertainties in the state matrices that are of norm-bounded type and satisfy $\Delta A_{1}, \Delta A_{2}, \Delta A_{d 1}, \Delta A_{d 2}=$

$H F\left[\begin{array}{lllll}E_{1} & E_{2} & E_{d 1} & E_{d 2}\end{array}\right] ; \alpha(\gamma)$ and $\beta(\delta)$

are variable time-lags in horizontal and vertical direction, respectively. Suppose $\alpha(\gamma)$ and $\beta(\delta)$ satisfy

$$
\begin{gathered}
\alpha_{1} \leq \alpha(\gamma) \leq \alpha_{h}, \beta_{1} \leq \beta(\delta) \leq \beta_{h}, \\
(3.51 \mathrm{c})
\end{gathered}
$$

where $\alpha_{1}$ and $\beta_{1}$ are positive constant integers symbolizing the lower time-lag limits in horizontal and vertical directions, respectively; $\alpha_{k}$ and $\beta_{k}$ are positive invariant integers symbolizing the upper time-lag limits in horizontal and vertical directions, respectively. It is presumed (Chen, 2010b; Tadepalli et al., 2016; Xu \& Yu, 2009) that system (3.51) has limited set of boundary conditions, that is $K$ and $L$ two nonnegative integers exist such that

$$
\begin{aligned}
& \chi(\gamma, \delta)=0, \forall y \geq K, \delta=-\beta_{h}, \beta_{h}+1_{s, \ldots, 0} \\
& \chi(\gamma, \delta)=u_{\mathrm{v} \sigma}, \forall 0 \leq K, \delta=-\beta_{h}, \beta_{h}+1_{, \ldots, 0}, \\
& \chi(\gamma, \delta)=0, \forall \delta \geq L, \delta=-\alpha_{h}, \alpha_{h}+1_{s, n} 0, \\
& \chi(\gamma, \delta)=\mathbf{0}, \forall \gamma \geq K, \delta=-\beta_{h}, \beta_{h}+1_{v} \ldots, 0, \\
& \chi(\gamma, \delta)=v_{\eta \sigma}, \forall 0 \leq \delta<L_{v} \gamma=-\alpha_{h},-\alpha_{h}+1_{v, \ldots}, 0_{x} \\
& u_{00}=v_{00^{x}} \\
& \text { (3.52) }
\end{aligned}
$$

\section{Theorem 3.3:}

Given positive integers $\alpha_{1}, \alpha_{h}, \beta_{1}, \beta_{h} \quad$ satisfying $0<\alpha_{1}<\alpha_{h}, 0<\beta_{1}<\beta_{h x}$ the system represented by (3.51)-(3.52) is globally asymptotically stable if there exist matrices $\quad \boldsymbol{P}_{i}>\mathbf{0}(i=1,2), \boldsymbol{Q}_{i}>0(i=1,2, \ldots \ldots, 6)$, $Z_{i}>0(i=1,2,3,4)$, matrices $S_{i}(i=1,2)$ with compatible dimensions, $\alpha_{i j}=\alpha_{j i}>0, \beta_{i j}=\beta_{j i}>0, g_{i}>0, i, j=1,2, \ldots, n(i \neq j)$ and $\epsilon>0$ such that the following LMIs hold

$$
\overline{\boldsymbol{\theta}}=\left[\begin{array}{cc}
Z_{2} & S_{1} \\
* & Z_{2}
\end{array}\right] \geq \mathbf{0} \quad \overline{\boldsymbol{\theta}}=\left[\begin{array}{cc}
Z_{4} & S_{2} \\
* & Z_{4}
\end{array}\right] \geq 0
$$$$
\left[\begin{array}{cccccc}
\Gamma_{11}+\epsilon E_{1}^{T} E_{1} & \epsilon E_{1}^{T} E_{2} & \epsilon E_{1}^{T} E_{d_{1}} & \epsilon E_{1}^{T} E_{d_{2}} & Z_{1} & 0 \\
* & \Gamma_{22}+\epsilon E_{2}^{T} E_{2} & \epsilon E_{2}^{T} E_{d_{1}} & \epsilon E_{2}^{T} E_{d_{2}} & 0 & 0 \\
* & * & \Gamma_{2 a}+\epsilon E_{d_{1}}^{T} E_{d_{1}} & \epsilon E_{d_{1}}^{T} E_{d_{2}} & Z_{2} S_{1}^{T} & -S_{1}+Z_{2} \\
* & * & * & \Gamma_{44}+\epsilon E_{d_{2}}^{T} E_{d_{2}} & 0 & 0 \\
* & * & * & * & Q_{1}-Z_{1}-Z_{2} & S_{1} \\
* & * & * & * & * & -Q_{2}-Z_{2} \\
* & * & * & * & * & * \\
* & * & * & * & * & * \\
* & * & * & * & * & * \\
* & * & * & * & * & * \\
* & * & * & * & * & *
\end{array}\right.
$$$$
\left.\begin{array}{ccccc}
0 & 0 & \Gamma_{19} & A_{1}^{T} G & 0 \\
Z_{2} & 0 & \Gamma_{29} & A_{2}^{T} G & 0 \\
0 & 0 & 0 & A_{d_{1}}^{T} G & 0 \\
Z_{4}-S_{2}^{T} & -S_{2}+Z_{4} & 0 & A_{d_{2}}^{T} G & 0 \\
0 & 0 & 0 & 0 & 0 \\
0 & 0 & 0 & 0 & 0 \\
-Q_{4}-Z_{2}-Z_{4} & S_{2} & 0 & 0 & 0 \\
* & -Q_{5}-Z_{4} & 0 & 0 & 0 \\
* & * & \Gamma_{99} & 0 & 0 \\
* & * & * & -G & -G H \\
* & * & * & * & -E I
\end{array}\right]<0
$$

where

$\Gamma_{11}=P_{1}+Q_{1}+Q_{2}+Q_{a}+\propto_{h \mathrm{~h}} Q_{a}-\Gamma_{19}-Z_{1}$

(3.55)

$\Gamma_{19}=\alpha_{1}^{2} Z_{1}-\alpha_{h 1}^{2} Z_{2}$

(3.56)

$\Gamma_{22}=-P_{2}+Q_{4}+Q_{5}+Q_{6}+\beta_{h 1} Q_{6} \Gamma_{29}-Z_{a}$

(3.57)

$\Gamma_{29}=-\beta_{0}^{2} Z_{a}-\beta_{h}^{2} Z_{4}$

(3.56)

$\Gamma_{\mathrm{a} a}=-Q_{a}-2 Z_{2}+S_{1}^{T}+S_{1}$

(3.59)

$\Gamma_{44}=-Q_{6}-2 Z_{4}+S_{2}^{T}+S_{2}$

(3.60)

$\Gamma_{99}=P_{1}-\Gamma_{19}+P_{2}-\Gamma_{29}-G_{i}^{*}$

(3.61)

Proof

Consider a 2-D Lyapunov functional (Tadepalli et al., 2016) $V(\chi(\gamma, \delta))=\bar{V}(\chi(\gamma, \delta))+\hat{V}(\chi(\gamma, \delta))$

$$
\bar{V}(\chi(\gamma, \delta))=\chi^{T}(\gamma, \delta) P_{1} \chi(\gamma, \delta)+\sum_{\gamma=-\varkappa_{1}}^{-1} \chi^{T}(\gamma+r, \delta) Q_{1} \chi(\gamma+r, \delta)
$$

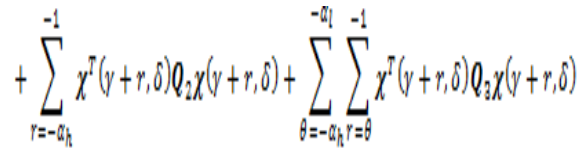

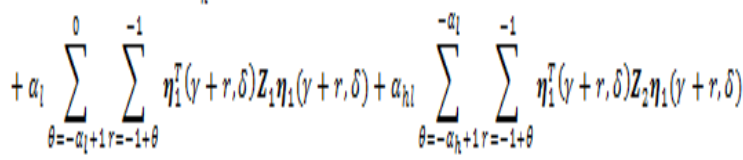




$$
\hat{V}(\chi(\gamma, \delta))=\chi^{T}(\gamma, \delta) P_{2} \chi(\gamma, \delta)+\sum_{\gamma=-\beta_{1}}^{-1} \chi^{T}(\gamma+r, \delta) Q_{4} \chi(\gamma+r, \delta)
$$

$+\sum_{\gamma=-\beta_{h}}^{-1} \chi^{T}(\gamma+r, \delta) Q_{5} \chi(\gamma+r, \delta)+\sum_{\theta=-\beta_{h}}^{-\beta_{1}} \sum_{\gamma=\theta}^{-1} \chi^{T}(\gamma+r, \delta) Q_{6} \chi(\gamma+r, \delta)$

$+\beta_{1} \sum_{\theta=-\beta_{\mathrm{l}}+1}^{0} \sum_{\gamma=-1+\theta}^{-1} \eta_{2}^{T}(\gamma+r, \delta) Z_{\mathrm{a}} \boldsymbol{\eta}_{2}(\gamma+r, \delta)$

$+\beta_{h \mathbb{l}} \sum_{\theta=-\beta_{h}+1}^{-\beta \eta} \sum_{r=-1+\theta}^{-1} \eta_{2}^{T}(\gamma+r, \delta) Z_{4} \eta_{2}(\gamma+r, \delta)$

and

$\eta_{1}(\gamma, \delta+1)=\chi(\gamma+1, \delta+1)-\chi(\gamma, \delta+1)=$

$\boldsymbol{f}(\psi(\gamma, \delta))-\chi(\gamma, \delta+1)$

$\eta_{2}(\gamma, \delta+1)=\chi(\gamma+1, \delta+1)-\chi(\gamma, \delta+1)=$

$\boldsymbol{f}(\psi(\gamma, \delta))-\chi(\gamma, \delta+1)$

(3.65b)

Taking the finite difference of LKF along the flight path of the system (3.51)-(3.52) and following the steps similar to (3.21)-(3.30) along the horizontal and vertical directions one can easily derive Theorem 3.3.

\section{Remark 3.3:}

The number of decision variables for Theorem 3.3 is given by $9 n^{2}+6 n+2$. It may be noted that as all the criteria presented are delay-dependent. And it is well known that the criteria depending on time-lag are less restrictive but have a computational overhead. So, the count of decision variables is an important issue.

\section{EXAMPLES}

For the purpose of comparative analysis consider the system (3.8)-(3.10b) with the following parameters (Kandanvli \& Kar, 2008).

$A=\left[\begin{array}{cc}0.8 & 0 \\ 0.5 & 0.9\end{array}\right], A_{d}=\left[\begin{array}{cr}0.01 & 0 \\ 0 & 0.2\end{array}\right]$.

$H=\left[\begin{array}{c}0 \\ 0.1\end{array}\right], E_{0}=\left[\begin{array}{ll}0.01 & 0\end{array}\right], E_{1}=\left[\begin{array}{ll}0 & 0.01\end{array}\right]$

$L=-1$

(4.68)

Our intent is to test the global asymptotic stability of the system described by (3.8)-(3.10). To achieve this we fix the lower delay bound $d_{1}$ and iteratively solve for the upper delay bound $d_{2}$ using Theorem 3.1 .

Using MATLAB, SeDuMi 1.21 solver (Sturm, 1999) and YALMIP 3.0 parser (Lofberg, 2004), the delay range over which Theorem 3.1 is capable of testing the system stability is $2 \leq d(r) \leq 23$. It may noted that the Theorem 3 in

\section{Example 4.1}

(Kandanvli \& Kar, 2008) is also able to evaluate the stability of the system under consideration for the same time-lag range.

Example 4.2 Consider an iterative control technique for batch procedures (Tadepalli et al., 2015). As shown in (Tadepalli et al., 2015) under a suitable control law and finite wordlength implementation a batch process can be expressed in the from of (3.31)-(3.37) with

$$
\begin{aligned}
A & =\left[\begin{array}{ccc}
0.2 & -0.1 & 0.09 \\
-0.23 & 0 & 0 \\
0.4 & -0.11 & 0.5
\end{array}\right], A_{d}=\left[\begin{array}{ccc}
0.3 & 0.1 & 0 \\
-0.17 & 0 & 0 \\
-0.25 & -0.2 & 0
\end{array}\right] \\
H & =\left[\begin{array}{cc}
0.01 & 0.01 \\
0 & 0 \\
-0.01 & -0.1
\end{array}\right], E_{0}=E_{1}=\left[\begin{array}{lll}
1 & 0 & 0 \\
0 & 1 & 0
\end{array}\right]
\end{aligned}
$$

$d_{1}^{\mathrm{h}}=3, d_{1}^{\mathrm{V}}=2, d_{2}^{\mathrm{V}}=24$

$(4.69 \mathrm{c})$

$L=1$

(4.69d)

Now, our objective is to evaluate the stability of the above system (which is under the influence of saturation finite word length nonlinearities) by iteratively solving for $d_{2}^{\text {hat }}$. With the help of Theorem 3.2 we find that the system mentioned above is stable all through the time-lag range $3 \leq d^{h}(\kappa) \leq 9$ and $2 \leq d^{v}(\lambda) \leq 24$.

Similarly, when $L=-1$ i.e. the system employs two's complement overflow correction the system is found to be stable over the time-lag range $3 \leq d^{h}(r) \leq 6$ and $2 \leq d^{v}(s) \leq 24$.

Next, we demonstrate the applicability of Theorem 3.3.

Example 4.3 Consider a class of systems described by Darboux equation. Darboux equation can be used to characterize thermic reactions in chemical processes, heat exchange process in car radiators, pipeline furnaces etc. As shown in (Tadepalli et al., 2016) the system described by Darboux equation can be modeled in the form of (3.51)-(3.52). Now, for the following parameters.

$$
\begin{aligned}
& A_{1}=\left[\begin{array}{ll}
0 & 1 \\
0 & 0
\end{array}\right], A_{2}=\left[\begin{array}{cc}
0 & 0 \\
0.25 & 0.25
\end{array}\right], A_{d_{1}}=\left[\begin{array}{ll}
0 & 0 \\
0 & 0
\end{array}\right], A_{d_{2}}= \\
& {\left[\begin{array}{ll}
0 & 0 \\
0 & -0.12
\end{array}\right],} \\
& H=\left[\begin{array}{c}
0 \\
0.1
\end{array}\right], E_{1}=E_{2}=\left[\begin{array}{ll}
0.01 & (4.70 a)
\end{array}\right], E_{d_{1}}=E_{d_{2}}\left[\begin{array}{ll}
0 & 0.01
\end{array}\right]
\end{aligned}
$$

$\alpha_{1}=3, \alpha_{\mathfrak{h}}=7, \beta_{\mathbb{l}}=2$

(4.70c)

$L=-1$

(4.70d) 
By determining the stability of the system using Theorem 3.3, we find that the system under consideration is stable over the time-lag range $3 \leq \alpha(\gamma) \leq 7$ and $2 \leq \beta(\delta) \leq 17$.

Further, the results obtained in examples 4.1-4.3 can be easily verified with the help of state trajectories which we are ignoring for the sake of brevity.

It may mentioned that Theorem 3.2 and Theorem 3.3 are significant in the sense that previously no delay-dependent results have been reported for 2-D discontinuous systems modeled by the Roesser and FMSLSS model under the simultaneous influence of generalized overflow nonlinear effects, uncertainties and variable time-lags.

\section{CONCLUSION}

This paper presents three time-lag contingent stability conditions in the form of Theorems 3.1-3.3. Although the highlight of this work is the stability analysis of 2-D discrete system characterized by the Roessser and FMSLSS models, but Theorem 3.1 is also significant in terms of smaller number of decision variables required while maintaining the same level of conservativeness in comparison with previously reported criterion. We demonstrated the applicability of Theorems 3.2 and 3.3 by considering the practical systems described in examples 4.2 and 4.3.

\section{REFERENCES}

1. Boyd, S., El Ghaoui, L., Feron, E., \& Balakrishnan, V. (1994). Linear matrix inequalities in system and control theory (Vol. 15). SIAM, Philadelphia, PA.

2. Chen, S.F. (2010a), Delay-dependent stability for 2D systems with time-varying delay subject to state saturation in the Roesser model. Applied Mathematics and Computation, 216(9), 2613-2622.

3. Chen, S.F. (2010b). Stability analysis for 2-D systems with interval time-varying delays and saturation nonlinearities. Signal Processing, 90(7), 2265-2275.

4. Dey, A., \& Kar, H. (2011a). LMI-based criterion for the robust stability of 2D discrete state-delayed systems using generalized overflow nonlinearities. Journal of Control Science and Engineering, 2011, 23.

5. Dey, A., \& Kar, H. (2011b). Robust stability of 2-D discrete systems employing generalized overflow nonlinearities: An LMI approach. Digital Signal Processing, 21(2), 262-269.

6. Fornasini, E., \& Marchesini, G. (1978). Double-indexed dynamical systems: State-space models and structural properties. Mathematical systems Theory, 12(1)59-72.

7. Huang, H., \& Feng, G. (2010). Improved approach to delay-dependent stability analysis of discrete-time systems with time varying delay. IET Control Theory \& Applications, 4(10), 2152-2159.

8. Kandanvli, V.K.R., \& Kar, H. (2008). Robust stability of discrete-time state-delayed systems employing generalized overflow nonlinearities. Nonlinear analysis: theory, Methods \& Applications, 69(9), 2780-2787.

9. Kandanvli, V.K.R., \& Kar, H. (2012). A delay-dependent approach to stability of uncertain discrete-time state-delayed systems with generalized overflow nonlinearities. ISRN Computational Mathematics, 2012.

10. Liu, D. (1998). Lyapunov stability of two-dimensional digital filters with overflow nonlinearities. IEEE Transactions on Circuits and Systems I: Fundamental Theory and applications, 45(5), 574-577.

11. Liu, J., \& Zhang, J. (2012). Note on stability of discrete time varying delay systems. IET Control Theory and Applications, 6(2), 335-339.

12. Lofberg, J.(2004). YALMIP: A toolbox for modeling and optimization in MATLAB. In Proceedings of Computer Aided Control Systems Design Conference, Taipei, Taiwan, 284-289.

13. Park, P., Ko, J. W., \& Jeong, C. (2011). Reciprocally convex approach to stability of systems with time varying delays. Automatica, 47(1), 235-238

14. Roesser, R. (1975). A discrete state-space model for linear image processing. IEEE Transactions on Automatic Control, 20(1), 1-10.
15. Sturm, J.F. (1999). Using SeDuMi 1.02, a MATLAB toolbox for optimization over symmetric cones. Optimization Methods and Software, 11(1-4), 625-653.

16. Tadepalli, S. K., Kandanvli, V.K.R., \& Kar, H. (2014). Stability criteria for uncertain discrete time systems under the influence of saturation nonlinearities and time varying delay. ISRN Applied Mathematics, 2014, 861759.

17. Tadepalli, S. K., \& Kandanvli, V.K.R. (2016). Improved stability results for uncertain discrete time state delayed systems in the presence of nonlinearities. Transactions of the Institute of Measurement and Control, 38(1), 33-43.

18. Tadepalli, S. K., \& Kandanvli, V.K.R. (2015). A new delay-dependent stability criterion for uncertain 2-D discrete systems described by Roesser model under the influence of quantization/overflow nonlinearities. Circuits, Systems, and Signal Processing, 34(8), 2537-2559.

19. Nurzaman, Tri Susilowati, and Arif Dian Wahyudi. "Drug Inventory Information System In Asy-Syifaa Yukum Jaya Islamic Hospital." International Journal of Communication and Computer Technologies 7.2 (2019), 27-31. Print. doi:10.31838/ijccts/07.02.07

20. R, karthik., Miruthula. A, and Nitheeswari. N. "Web Based Online Machine Controlling And Monitoring Using PLC Via Modbus Communication." International Journal of Communication and $\begin{array}{lllll}\text { Computer Technologies } 7.2 & \text { (2019), 22-26. Print }\end{array}$ doi:10.31838/ijccts/07.02.06

21. Rao, B. Venkateswara, et al. "Optimal power flow by Newton method for reduction of operating cost with SVC models." 2009 International Conference on Advances in Computing, Control, and Telecommunication Technologies. IEEE, 2009.

22. Xu, J., \& Yu, L. (2009). Delay dependent guaranteed cost control for uncertain 2-D discrete systems with state delay in the FM second model. Journal of the Franklin Institute, 346(2), 159-174 